Astrolabio. Revista internacional de filosofía

Año 2018 Núm. 22. ISSN 1699-7549. pp. 239-251

doi: $10.1344 /$ astrolabio2018.22.20

\title{
La reivindicación feminista del derecho a la salud
}

\section{Feminist Vindication of the Right to Health}

\author{
Tasia Aránguez Sánchez
}

\begin{abstract}
Resumen: Desde los años noventa del siglo pasado se está produciendo dentro de la medicina una lenta transformación que cuestiona el androcentrismo de dicha ciencia. Los aspectos cuestionados conciernen a los estereotipos de género presentes en la atención sanitaria, la exclusión de las mujeres de los ensayos clínicos, o la falta de conocimiento suficiente sobre el funcionamiento del cuerpo de las mujeres. Uno de los aspectos de esta transformación es el creciente interés por las enfermedades de alta prevalencia femenina como la fibromialgia y la endometriosis. Las asociaciones de mujeres afectadas reivindicamos nuestro derecho a la salud que todavía no está siendo garantizado de forma efectiva. Las enfermedades son muchas veces confundidas con dolencias de tipo psicosomático y el diagnóstico se demora durante años, con muchos especialistas consultados. Apenas se invierten recursos en estas enfermedades y, como consecuencia, las técnicas de diagnóstico son pobres y los tratamientos insuficientes. A esto se suman las extraordinarias dificultades para el acceso a pensiones de la seguridad social que enfrentan las afectadas cuando la enfermedad es incapacitante. Dichas dificultades son producto de la falta de perspectiva de género de la legislación en materia de seguridad social. El mainstreaming de género es la exigencia legal de incorporar esta perspectiva a las políticas públicas y a las normas, lo que habría de conducir a transformaciones en el ámbito sanitario.
\end{abstract}

Palabras clave: Derecho a la salud, mujeres, feminismo, mainstreaming

Abstract: Since the nineties of the last century a slow transformation is taking place in medicine field that questions the androcentrism of this science. The questioned aspects concern the gender stereotypes present in health care, the exclusion of women from clinical trials, or the lack of sufficient knowledge about the functioning of the women's body. One of the aspects of this transformation is the growing interest in diseases that mainly affect women such as fibromyalgia and endometriosis. The associations of affected women claim our right to health, which is not yet being guaranteed effectively. The diseases are often confused with psychosomatic diseases and the diagnosis is delayed for years, with many specialists consulted. Resources are hardly invested in these diseases and, as a consequence, diagnostic techniques are poor and treatments are insufficient. Added to this are the extraordinary difficulties for accessing benefits for disability (when these diseases are disabling). These difficulties are the product of the lack of a gender perspective in the social security law. Gender mainstreaming is the legal requirement to incorporate this perspective into public policies and norms, which would lead to transformations in the health field.

Keywords: Right to health, women, feminism, mainstreaming 
Astrolabio. Revista internacional de filosofía

Año 2018 Núm. 22. ISSN 1699-7549. pp. 239-251

doi: $10.1344 /$ astrolabio2018.22.20

\section{INTRODUCCIÓN:}

\section{EL DESARROLLO DE LA PERSPECTIVA DE GÉNERO EN LA MEDICINA}

En los años setenta del siglo pasado el feminismo desarrolló una crítica al androcentrismo científico ${ }^{1}$ pero dicha crítica no movió los pilares de la medicina hasta los años noventa. Las enfermedades de mujeres y otras diferencias sanitarias no comenzaron a estudiarse hasta los años ochenta porque, como sostiene Carme Valls ${ }^{2}$, hasta ese momento se sostenía que hombres y mujeres son iguales en lo biológico y que las diferencias entre ambos sexos son exclusivamente sociales. Cuando las diferencias comenzaron a estudiarse el enfoque dominante fue el de erradicar hormonalmente los fenómenos exclusivos de las mujeres como la menstruación y la menopausia (pues se partía del modelo masculino y lo propio de las mujeres se veía como problema de salud). Como indica Isabel Ruiz Pérez $z^{3}$, en los años noventa se denunció el desconocimiento científico acerca de la salud de las mujeres (que va mucho más allá de la salud de reproductiva) y comenzó el interés por las enfermedades de alta prevalencia femenina. En los noventa se produjo un cuestionamiento de la investigación y las pruebas de medicamentos, por la ausencia de participación femenina en dichos estudios. En aquella década, por tanto, se produjo un fuerte desarrollo de la perspectiva de género en la medicina que hoy continúa desarrollándose. A pesar de esto, como veremos, todavía queda mucho por hacer para que logremos la igualdad en el derecho a la salud.

La nueva perspectiva de la salud considera que son relevantes tanto las diferencias de género como las diferencias de sexo, es decir, tanto las sociales como las fisiológicas. Los aspectos sociales de la salud femenina tienen en cuenta cuestiones como la incidencia de los estereotipos de género sobre la atención sanitaria y los déficits en la investigación de la salud de las mujeres. En el derecho español la perspectiva de género se ha integrado expresamente en la salud a través de la Ley para la Igualdad Efectiva de Mujeres y Hombres (Ley orgánica 3/2007) establece la obligación de integrar el principio de igualdad en investigaciones y programas de salud: «Las políticas, estrategias y programas de salud integrarán activamente en sus objetivos y actuaciones el principio de igualdad entre mujeres y hombres, evitando que, por sus diferencias físicas o por los estereotipos sociales asociados, se produzcan discriminaciones entre ellos en los objetivos y actuaciones sanitarias» ${ }^{4}$.

\footnotetext{
${ }^{1}$ Mozo González, C. (2006). "Construcción social del género y su impacto en la salud”. En Actas de las Jornadas Andaluzas Mujeres y Salud. Mirando la Salud desde una perspectiva de género. Málaga: Instituto Andaluz de la Mujer. Consejería para la igualdad y bienestar social.

2 Valls-Llobet, C. (2006). "El derecho de las mujeres a conocer, decidir y ser protagonistas de su salud" En Actas de las Jornadas Andaluzas Mujeres y Salud. Mirando la Salud desde una perspectiva de género. Málaga: Instituto Andaluz de la Mujer. Consejería para la igualdad y bienestar social.

${ }^{3}$ Ruiz Pérez, I. (2006). "Sesgo de género en los estudios con medicamentos en las mujeres". En Actas de las Jornadas Andaluzas Mujeres y Salud. Mirando la Salud desde una perspectiva de género. Málaga: Instituto Andaluz de la Mujer. Consejería para la igualdad y bienestar social.

${ }_{4}^{4}$ Ley 14/1986 General de Sanidad, artículo 3.4. Añadido por la Ley Orgánica 3/2007 para la igualdad efectiva de mujeres y hombres, a través de la disposición adicional octava.
} 
Astrolabio. Revista internacional de filosofía Año 2018 Núm. 22. ISSN 1699-7549. pp. 239-251

doi: 10.1344/astrolabio2018.22.20

\section{DESIGUALDADES DE GÉNERO EN LAS POLÍTICAS SANITARIAS}

Ann Hohmann ${ }^{5}$ demostró que las mujeres recibían más ansiolíticos y antidepresivos que los hombres y Colameco ${ }^{6}$ aporta estudios que demuestran que los médicos de ambos sexos aprecian a menudo que los problemas de salud de los pacientes varones son más graves y estiman con más frecuencia que se trata de un problema psicológico si la paciente es mujer.

Los estereotipos de género están detrás de estas desigualdades. Por ejemplo, hoy se sabe que las mujeres padecen patologías crónicas con más frecuencia que los hombres, mientras que estos presentan mayores índices de patologías agudas. Por eso las mujeres utilizan los servicios de atención primaria con más frecuencia que los hombres, expresando cansancio y dolor crónico. Como señala Valls Llobet antes de los noventa la mayoría de los epidemiólogos sostenían que las mujeres acudían a los ambulatorios porque tenían más tiempo libre hasta que los estudios de medicina diferencial demostraron que las mujeres

«no iban porque tuvieran ganas de pasear, sino porque querían ser atendidas del problema de cansancio o dolor que tenían.»

Estos estereotipos operan generando dos situaciones discriminatorias:

«A la mujer que tiene un problema biológico se le dice que es psicológico y a la que tiene un problema social se le dice también que es psicológico y no se indagan las verdaderas causas de la enfermedad». ${ }^{8}$

La desigualdad no solo se produce en la atención sanitaria sino también en la investigación.

Hasta los años noventa del siglo pasado la mayoría de los conocimientos científicos que se utilizaban para tomar decisiones sobre diagnóstico y tratamiento se basaban en estudios realizados solo en hombres ${ }^{9}$. Desde entonces ha aumentado la información recopilada sobre la salud de las mujeres. Sin embargo, esta situación aún no ha mejorado demasiado ya que los ensayos clínicos solo incluyen un $24,6 \%$ de mujeres y solo se ha realizado un

\footnotetext{
${ }^{5}$ Hohmann, A. (1989). "Gender Bias in Psychotropic Drug Prescribing in Primary Care”. Medical Care. Philadelphia: University of Massachusetts Medical School

${ }^{6}$ Colameco,S; Becker, L.A.; Simpson, M. (1983). "Sex bias in the assessment of patients complaints". J-Fam-Pract. 16, Oxford: Oxford University Press, pp. 1117-1121

7 Valls-Llobet, C. (2006). "El derecho de las mujeres a conocer, decidir y ser protagonistas de su salud". En Actas de las Jornadas Andaluzas Mujeres y Salud. Mirando la Salud desde una perspectiva de género. Málaga: Instituto Andaluz de la Mujer. Consejería para la igualdad y bienestar social. p. 61.

${ }^{8}$ Ibid. p.62.

9 Valls-Llobet, C. (2011). "Morbilidad diferencial entre mujeres y hombres". Feminismo/s 18, Alicante: Instituto Universitario de Estudios de Género, pp. 281-290
} 
Astrolabio. Revista internacional de filosofía Año 2018 Núm. 22. ISSN 1699-7549. pp. 239-251

doi: $10.1344 /$ astrolabio2018.22.20

análisis especifico de género en un $14 \%$ de los ensayos ${ }^{10}$. Isabel Ruiz Pérez ${ }^{11}$ señala que entre los medicamentos con sesgos de género en los ensayos se encuentran algunos tan importantes como los antibióticos, los antiarrítmicos cardiacos, los antirretrovirales, y los antipsicóticos. No hay suficientes datos acerca de cómo afectan estos medicamentos a las mujeres, en interacción con la menstruación, el embarazo o los anticonceptivos. El déficit de conocimiento sobre los efectos secundarios en las mujeres es uno de los motivos por los que, según Ruiz Pérez, se tiende a prescribir a las mujeres más terapias paliativas o alternativas. Y eso no siempre es positivo para resolver el problema de salud.

Junto con los ámbitos de la atención sanitaria y la investigación también resultan problemáticas la organización y estructura de los servicios de asistencia sanitaria. La medicina ha desarrollado en los últimos treinta años un proceso de superespecialización ${ }^{12}$. Cada parte del cuerpo es tratada por separado, con poco diálogo entre disciplinas médicas y escasos equipos multidisciplinares. Esto perjudica a las enfermedades con base endocrina y repercusión en todo el cuerpo, que son prevalentes entre las mujeres. Otro aspecto relevante del androcentrismo sanitario es la falta de respeto hacia el cuerpo de las mujeres que se refleja en el frecuente desconocimiento de las funciones que tienen la menstruación y los órganos reproductivos sobre el sistema endocrino de las mujeres y su salud general. Como señala Valls Llobet, «la falta de respeto por el cuerpo de las mujeres, está conduciendo a las propuestas de abolición bioquímica de la menstruación» ${ }^{13}$, la endocrinóloga señala la repercusión de la ovulación sobre la salud musculoesquelética, y el equilibrio de los neurotransmisores y critica que se traten la menstruación y la ovulación como si solo fuesen relevantes para la reproducción. Todavía parece perpetuarse la vieja idea de que la menstruación es una fuente de problemas que debe eliminarse. Esto se plasma en la enorme facilidad con la que a las mujeres se les paraliza la ovulación o se les quita el útero, las trompas o los ovarios como soluciones terapéuticas.

\section{ENFERMEDADES DE ALTA PREVALENCIA FEMENINA Y POLÍTICAS PÚBLICAS}

Las enfermedades de alta prevalencia femenina son aquellas que se presentan con mayor frecuencia en mujeres que en hombres. Como señala Carme Valls-Llobet ${ }^{14}$ algunas de ellas son exclusivas de mujeres (como la endometriosis, los tumores ginecológicos y las

\footnotetext{
10 Valls-Llobet, C. (2006). "La menstruación: de la invisibilidad a la abolición”. Duoda. Estudis de la Diferencia Sexual. num 31, Barcelona: Universitat de Barcelona, p. 74.

${ }^{11}$ Ruiz Pérez, I. (2006). "Sesgo de género en los estudios con medicamentos en las mujeres". En Actas de las Jornadas Andaluzas Mujeres y Salud. Mirando la Salud desde una perspectiva de género. Málaga: Instituto Andaluz de la Mujer. Consejería para la igualdad y bienestar social.

12 Valls-Llobet, C. (2006). "La menstruación: de la invisibilidad a la abolición". Revista Duoda, 31. Barcelona: Universitat de Barcelona, op. cit.

${ }^{13}$ Valls-Llobet, C. (2003). "Morbilidad invisible y cooperación”. Quadern CAPS, op. cit. p. 83.

14 Valls-Llobet, C. (2001). "Desigualdades de género en Salud Pública”. Quadern CAPS, n’. 30.
} 
Astrolabio. Revista internacional de filosofía

Año 2018 Núm. 22. ISSN 1699-7549. pp. 239-251

doi: $10.1344 /$ astrolabio2018.22.20

enfermedades derivadas de embarazos y partos $^{15}$ ) y otras son padecidas por mujeres en una frecuencia mucho mayor (fibromialgia, anemias, dolor crónico, enfermedades autoinmunes, las enfermedades endocrinológicas, trastorno ansioso depresivo, enfermedades del tiroides, lupus, artritis reumatoide, Síndrome de sjögren, esclerodermia, miastenia gravis y diabetes mellitus autoinmune ${ }^{1617}$. Las enfermedades de alta prevalencia femenina se caracterizan por estar asociadas a estereotipos sobre las mujeres y por recibir una atención menor. La endometriosis representa un caso claro del androcentrismo en la investigación científica, pues a pesar de que es una enfermedad que afecta a más de 170 millones de mujeres (más de dos millones de mujeres en España), la enfermedad no despierta apenas interés científico. Como señala el ginecólogo Carmona ${ }^{18}$ (reconocido especialista en la enfermedad), «Es una enfermedad tan frecuente que ni siquiera sabemos el porcentaje real de afectadas». Se calcula que la padecen entre $10 \%$ y el $15 \%$ de las mujeres, «quizás incluso el 20\%». Para que podamos ponderar el alcance de esta invisibilidad resulta útil saber que En EEUU los Institutos Nacionales de Salud financian los estudios sobre diabetes, una enfermedad que afecta a cerca de 14 millones de mujeres, con 100 veces más dinero que los que se hacen sobre endometriosis, que afecta a unos 8 millones de mujeres en ese país ${ }^{19}$. El resultado es la escasez de investigación, la escasez de especialistas y la falta de formación en atención primaria. Las técnicas de diagnóstico son pobres y el diagnóstico definitivo requiere una cirugía, por lo que solo los estadios avanzados de la enfermedad en los que el dolor es insoportable se exponen a una laparoscopia diagnóstica. Los tratamientos farmacológicos que se ofrecen a las afectadas fueron diseñados para otros fines (incluso uno de los más recetados ${ }^{20}$, para el tratamiento del cáncer de próstata).

La ausencia de formación en "morbilidad diferencial” (diferencias en el enfermar entre hombres y mujeres) fomenta, según Valls «la cultura del sufrimiento que intenta esconder la ignorancia científica de las causas del malestar y la sintomatología, predicando a las mujeres la resignación y la sumisión a un supuesto destino fatal que las obliga

\footnotetext{
15 Valls-Llobet, C. (2006). "La menstruación: de la invisibilidad a la abolición”. Duoda. Estudis de la Diferencia Sexual. num 31. pp. 71-84

16 Valls-Llobet, C. (2003). "Morbilidad invisible y cooperación”. Quadern CAPS, ISSN 0213-4462, No. 31, 2003. pp. 60-67

17 Según esta autora, por edades se pueden resaltar las siguientes enfermedades prevalentes: 14-45 años: anemias y ferropenias (las padecen el 75\% de la población femenina); las metrorragias (50\% de la población femenina), la amenorrea u oligomenorrea (de un 15 a un $20 \%$ de la población femenina), endometriosis (15 a 20\%) y el síndrome premenstrual severo (de un 15 a un 35\%); las hiperprolactinemias; los trastornos de la función tiroidea, como el hipertiroidismo, las tiroiditis autoinmunes, el hipotiroidismo clínico y subclínico; y las enfermedades autoinmunes. Más de 45: cáncer de mama, de cérvix y de endometrio; cardiopatía isquémica; diabetes clínica y subclínica; cáncer de colon y de pulmón; deficiencia de formación ósea por déficit subclínico de vitamina $\mathrm{D}$; hipertensión y las dislipemias; hipotiroidismo clínico y subclínico (15 a 20\% de mujeres), el hipertiroidismo ( $2 \%$ de mujeres), la angina inestable, el déficit subclínico de vitamina D (30\% de la población femenina), y el hiperparatiroidismo secundario (20\%).

18 Pinto, T. (2016). "La endometriosis, la enfermedad de las mujeres ninguneada por la sociedad". Eldiario.es. 12/03/2016.

${ }^{19} \mathrm{El}$ doctor Carmona expone estos datos.

${ }^{20}$ Hemos optado por no mencionar expresamente el nombre del medicamento, pues tal vez no sea adecuado en sede académica. No obstante, animamos a una pequeña indagación al respecto.
} 
Astrolabio. Revista internacional de filosofía

Año 2018 Núm. 22. ISSN 1699-7549. pp. 239-251

doi: 10.1344/astrolabio2018.22.20

a vivir con molestias y con dolor» ${ }^{21}$. Las patologías femeninas padecen un déficit en métodos diagnósticos y desarrollo de tratamientos específicos. La fibromialgia es frecuentemente "tratada" con antidepresivos y analgésicos. Hay médicos que consideran que las quejas de la mujer responden a un problema psicológico. Esta situación es compartida con otras enfermedades "invisibles" de alta prevalencia femenina cuyo síntoma principal es el dolor crónico, como la endometriosis. En el caso de la endometriosis, la coincidencia de muchos de sus síntomas con el periodo menstrual aumenta todavía más las posibilidades de que la mujer sea considerada hipocondriaca y exagerada, ya que la sociedad ha normalizado la idea de que la menstruación duele. Las quejas de dolor extremo por parte de las mujeres dan lugar a un largo periplo médico de ocho años de duración antes de obtener un diagnóstico consultando a cuatro o cinco especialistas y pasando por salud mental. En ese periodo la enfermedad se vuelve incapacitante y extremadamente difícil de operar, mientras atención primaria despacha a la mujer con analgésicos y antidepresivos. Isabel Ruiz Pérez ${ }^{22}$ formó parte de un estudio que puso de manifiesto que el hombre con fibromialgia está de baja laboral con mayor frecuencia que la mujer con idéntico diagnóstico (con una fibromialgia de la misma gravedad). Además, los hombres reciben con mayor frecuencia un tratamiento farmacológico para la enfermedad, mientras que en muchas ocasiones a las mujeres se les prescriben terapias alternativas.

La organización de la atención sanitaria, con su distribución por especialidades, no suele detectar enfermedades endocrinas tan complejas como la endometriosis o las enfermedades tiroideas. La endometriosis tiene muchos síntomas no ginecológicos como disquecia, estreñimiento, sangre en las heces, diarreas o dolor al orinar y otros ginecológicos (dismenorrea, aumento de la sintomatología durante la menstruación). Es usual que la mujer acuda a digestivo con unos síntomas y a ginecología con otros, sin que se produzca la necesaria sospecha clínica en ninguna de ambas consultas (que minusvaloran la situación al carecer del cuadro completo). Si la fragmentación del saber médico es un obstáculo para el diagnóstico, aún más lo es para el tratamiento quirúrgico. Las cirugías de endometriosis tienen una complejidad igual o superior a las oncológicas, pues el tejido invade numerosos órganos y eso hace necesaria la intervención de un equipo multidisciplinar. Es muy frecuente que la cirugía deje focos de endometriosis sin eliminar, de modo que estos se reproducen tras la cirugía y esta resulta inefectiva e incluso perjudicial, empeorando la enfermedad. Muchas afectadas acumulan tres o cuatro cirugías incompletas que les han ido dejado secuelas cumulativas. No todas las Comunidades Autónomas cuentan con un equipo multidisciplinar con competencia para abordar estas cirugías con éxito, de modo que es frecuente que las pacientes realicen un

\footnotetext{
21 Valls-Llobet, C. "Las causas orgánicas del malestar de las mujeres”. Mujeres y Salud. n 16. p. 6. Barcelona: Centro de Análisis y Programas Sanitarios

${ }^{22}$ Ubago Linares, M. C.; Plazaola Castaño, J.; Ruiz Pérez, I.; Olry de Labrylima, A.; Bermejo Pérez, M. J. (2005). "Características clínicas y psicosociales de personas con fibromialgia: repercusión del diagnóstico sobre sus actividades”. Revista española de salud pública, vol. 79, ñ. 6, (pp. 683-695); Ruiz Pérez, I.; Ubago Linares, M. C. (2004). "Fibromialgia: ¿un problema real o imaginario?”, Meridiam, $n^{0} .34$, (pp. 37-38)
} 
Astrolabio. Revista internacional de filosofía

Año 2018 Núm. 22. ISSN 1699-7549. pp. 239-251

doi: 10.1344/astrolabio2018.22.20

frustrante peregrinaje. Seguramente la escasez de especialistas en endometriosis está detrás de la frecuencia de histerectomías totales, pues es muy difícil eliminar todo el tejido invasor manteniendo la integridad del tejido sano (ambos están adheridos).

La invisibilidad a la que se enfrentan las enfermedades de alta prevalencia femenina se traslada al derecho sin cuestionamiento. Así, la endometriosis, la fibromialgia y otras de las citadas, no aparecen en el Real Decreto 1971/1999 que establece las enfermedades que son causa de reconocimiento de discapacidad. Es decir, a pesar de que esta es una norma con pretensión de exhaustividad, no aparecen en ella las enfermedades más incapacitantes para las mujeres como la fibromialgia. Esta ausencia dificulta mucho a las mujeres que padecen importantes limitaciones la consecución de un grado de discapacidad que les ofrezca protección jurídica frente a las consecuencias sociales de su discapacidad fáctica (por ejemplo, la nulidad de los despidos a causa de la enfermedad o los incentivos a la contratación). En la incapacidad permanente, a pesar de valorarse de un modo muy distinto (la judicatura juzga a partir de informes médicos si una persona concreta está incapacitada para realizar un trabajo específico), se percibe la misma situación de ostracismo. En un estudio que hemos realizado de 65 sentencias de incapacidad permanente por endometriosis ${ }^{23}$ observamos que cuando la endometriosis aparece en el cuadro clínico de una mujer entre otras enfermedades, lo más probable es que su presencia sea ignorada. Es decir, la enfermedad no es tenida en cuenta ni en la argumentación jurídica, ni en el fallo y se trata la enfermedad igual que si no apareciera. De modo que es como si no hubiera aparecido. Por tanto, el estudio viene a confirmar que la endometriosis padece una situación de invisibilidad jurídica y que las mujeres incapacitadas para el trabajo por la misma tienen grandes dificultades para acceder a una pensión. La situación no es achacable tan solo a la medicina dado que el antes aludido estudio de Ruiz Pérez ${ }^{24}$ refirió que, ante un mismo diagnóstico de fibromialgia, un hombre tiene más posibilidades de obtener una incapacidad permanente que una mujer. Esto pone de manifiesto que los estereotipos de género están presentes también en el estamento jurídico.

La situación expuesta de las enfermedades de alta prevalencia femenina ha dado lugar a una intensa labor reivindicativa por parte de asociaciones de mujeres afectadas

\footnotetext{
${ }^{23}$ En la gran mayoría de las 65 sentencias analizadas, la endometriosis aparece en el cuadro clínico, pero no vuelve a mencionarse en la argumentación jurídica. En muchas de ellas se dice expresamente cuáles de las enfermedades del cuadro clínico son más relevantes para valorar el caso. La endometriosis es la menos relevante de todas, seguida de la fibromialgia. Ejemplos de sentencias en los que la endometriosis aparece en el cuadro clínico pero no vuelve a mencionarse en la argumentación jurídica son las siguientes: STSJ/Murcia 2137/2016, de 10 de Octubre, STSJ/Galicia 5044/2016, de 7 de Julio, STSJ/País Vasco 1531/2016, de 10 de Mayo, STSJ/Madrid 4937/2016, de 25 de Abril, STSJ/Andalucía 1488/2016, de 14 de Marzo, STSJ/Valencia 6069/2015, de 30 de Octubre, STSJ/Oviedo 1741/2015, de 26 de Junio, STSJ/Cataluña 12199/2014, de 17 de Diciembre, STSJ/Andalucía 10556/2014, de 27 de Noviembre, STSJ/País Vasco 1096/2016 de 12 de Abril, STSJ/Galicia 1939/2016 de 18 de Marzo, STSJ/Galicia 10123/2015 de 11 de Diciembre, STSJ/Galicia 8721/2015 de 16 de Noviembre (entre otras muchas).

${ }^{24}$ Ubago Linares, M. C.; Plazaola Castaño, J.; Ruiz Pérez, I.; Olry de Labry Lima, A.; Bermejo Pérez, M. J. (2005). "Características clínicas y psicosociales de personas con fibromialgia: repercusión del diagnóstico sobre sus actividades". Revista española de salud pública, vol. 79, n. 6, pp. 683-695; Ruiz Pérez, I.; Ubago Linares, M. C. (2004). "Fibromialgia: ¿un problema real o imaginario?”, Meridiam, $\mathrm{n}^{\circ}$. 34, París: United Nations Principles for Responsible Investmen, pp. 37-38.
} 
Astrolabio. Revista internacional de filosofía

Año 2018 Núm. 22. ISSN 1699-7549. pp. 239-251

doi: $10.1344 /$ astrolabio2018.22.20

que claman por un igualitario acceso al derecho a la salud y por el igual acceso a las prestaciones de la seguridad social cuando la enfermedad se hace incapacitante. En esa lucha nos encontramos las afectadas de endometriosis. Estas demandas forman parte, como se ha expuesto, de una larga batalla histórica contra el androcentrismo en la medicina. Las políticas públicas habrían de comprometerse activamente con la igualdad en el ámbito sanitario y en la investigación médica, de acuerdo con el artículo 27 de la Ley Orgánica de Igualdad Efectiva de Mujeres y Hombres ${ }^{25}$ y con la estrategia de la ONU y la Unión Europea denominada "mainstreaming de género" ${ }^{26}$ que debe informar todas las políticas sanitarias. Normativas autonómicas, como el artículo 41 de la Ley 12/2007 de Promoción de la Igualdad de Género en Andalucía exigen expresamente que se impulse la prevención y el tratamiento de enfermedades de alta prevalencia femenina, citándose la anorexia, la bulimia y la fibromialgia. Una Declaración conjunta del Congreso de los Diputados de $2005^{27}$ expresó asimismo voluntad política de establecer consensualmente protocolos de diagnóstico y tratamiento de las distintas patologías que subyacen tras la fibromialgia y la fatiga crónica, formar a profesionales de la medicina sobre la enfermedad, fomentar la investigación en causas y tratamientos y elaborar protocolos de evaluación de las incapacidades para la profesión habitual. A pesar de tan encomiables promesas, la situación institucional de las afectadas por estas enfermedades apenas ha visto cambios.

\footnotetext{
25 Articulo 27 Integración del principio de igualdad en la politica de salud

1. Las políticas, estrategias y programas de salud integrarán, en su formulación, desarrollo y evaluación, las distintas necesidades de mujeres y hombres y las medidas necesarias para abordarlas adecuadamente.

2. Las Administraciones públicas garantizarán un igual derecho a la salud de las mujeres y hombres, a través de la integración activa, en los objetivos y en las actuaciones de la política de salud, del principio de igualdad de trato, evitando que por sus diferencias biológicas o por los estereotipos sociales asociados, se produzcan discriminaciones entre unas y otros.

3. Las Administraciones públicas, a través de sus Servicios de Salud y de los órganos competentes en cada caso, desarrollarán, de acuerdo con el principio de igualdad de oportunidades, las siguientes actuaciones:

a) La adopción sistemática, dentro de las acciones de educación sanitaria, de iniciativas destinadas a favorecer la promoción específica de la salud de las mujeres, así como a prevenir su discriminación.

b) El fomento de la investigación científica que atienda las diferencias entre mujeres y hombres en relación con la protección de su salud, especialmente en lo referido a la accesibilidad y el esfuerzo diagnóstico y terapéutico, tanto en sus aspectos de ensayos clínicos como asistenciales.

c) La consideración, dentro de la protección, promoción y mejora de la salud laboral, del acoso sexual y el acoso por razón de sexo.

d) La integración del principio de igualdad en la formación del personal al servicio de las organizaciones sanitarias, garantizando en especial su capacidad para detectar y atender las situaciones de violencia de género.

e) La presencia equilibrada de mujeres y hombres en los puestos directivos y de responsabilidad profesional del conjunto del Sistema Nacional de Salud.

f) La obtención y el tratamiento desagregados por sexo, siempre que sea posible, de los datos contenidos en registros, encuestas, estadísticas u otros sistemas de información médica y sanitaria.

${ }^{26}$ Plataforma para la acción de la cuarta Conferencia Mundial sobre la mujer. Naciones Unidas. Beijing. (1995). Punto cuatro: Los gobiernos y otros actores promocionarán una política activa y visible del mainstreaming de género, en todas las políticas y programas, para que, antes de que se tomen las decisiones, se realice un análisis de los efectos producidos en mujeres y hombres, respectivamente.

27 Boletín Oficial de las Cortes Generales de 27 de abril de 2005.
} 
Astrolabio. Revista internacional de filosofía

Año 2018 Núm. 22. ISSN 1699-7549. pp. 239-251

doi: $10.1344 /$ astrolabio2018.22.20

\section{EL MAINSTREAMING DE GÉNERO EN LAS POLÍTICAS SANITARIAS}

El mainstreaming de género es una estrategia de aplicación de las políticas de igualdad entre mujeres y hombres. Dicha estrategia, que ha sido impulsada por la ONU y posteriormente por la Unión Europea ${ }^{28}$, ha sido implementada en España con el nombre de "transversalidad". Una característica central ${ }^{29}$ del Mainstreaming es que la igualdad tiene que ser incorporada a todas las políticas (educación, transporte, salud, etc.), dado que una misma medida puede afectar a mujeres y hombres de modo distinto, generando una discriminación. El mainstreaming lo que implica es que las políticas deben ser una prioridad en la agenda política dominante, en las políticas generales. María Ángeles Barrère ${ }^{30}$ enfatiza otra característica del mainstreming que suele ignorarse, que es la elevación a corriente principal (esto es lo que significa la palabra) de la perspectiva de género. En efecto las mujeres sufren discriminación en todos los países del mundo. Aunque las mujeres no configuran un grupo homogéneo (hay diferencias de orientación sexual, etnia, clase, religión, etc.) es indudable que el sistema sexo-género tiene un papel fundamental en la desigualdad. Por eso el principio de mainstreaming no puede traducirse como mera transversalidad, porque entonces olvidamos que enfatiza el hecho de que el sistema sexo-género atraviesa los distintos sistemas de discriminación. El mainstreaming surge en el derecho internacional en la IV Conferencia Mundial sobre la Mujer celebrada en Beijing en 1995, si bien encuentra su precedente en la Conferencia de Derechos

\footnotetext{
${ }^{28}$ Esta estrategia fue asumida explícitamente por la Plataforma para la Acción de la Cuarta Conferencia Mundial sobre Mujeres de Naciones Unidas celebrada en Pekín en 1995: «Los gobiernos y otros actores promocionar una política activa y visible del mainstreaming de género, en todas las políticas y programas, para que, antes de que se tomen las decisiones, se realice un análisis de los efectos producidos en mujeres y hombres, respectivamente».

${ }^{29}$ Emmanuela Lombardo. El mainstreaming. La aplicación de la transversalidad en la Unión Europea. Explica otras características: -Incorporación de la perspectiva de género a la agenda política dominante: el mainstreaming implica que los problemas de igualdad tienen que ser tratados dentro de todas las políticas.

-Noción profunda de la igualdad de género: La igualdad de género que se busca no es de iure, sino de facto, y se pretende lograr mediante una perspectiva de género, y no un enfoque limitado a los problemas de mujeres. Esto implica utilizar un enfoque que combata de forma explícita el sistema patriarcal, prestando atención a la tupida red de desventajas que padecen las mujeres en la familia, el trabajo remunerado, la política, la sexualidad, la cultura, la violencia machista, la salud, etc.

-Participación de las mujeres en las instituciones políticas y en los procesos de toma de decisiones: la Decisión de la Comisión 2000/407 recomienda que la participación de las mujeres sea al menos del 40\%.

-Prioridad a las políticas de igualdad de género y a las de especial relevancia para las mujeres: en términos de recursos financieros, humanos y en el tipo de medidas adoptadas.

-Coordinación interdepartamental entre organismos e instituciones, y formación en perspectiva de género: la parcelación característica de la estructura burocrática de las instituciones puede dificultar la remoción de obstáculos a la discriminación. También es necesario incrementar la formación en igualdad de género para eliminar los prejuicios de la acción política.

-Incluir la variable de género en las políticas y estadísticas. Los datos estadísticos deben estar desagregados por género y los análisis coste-beneficio han de tener en cuenta el impacto diferencial en las mujeres y hombres, y sus respectivas necesidades.

-La adopción de decisiones políticas debe incluir no solo a los actores ordinarios de la política, sino también a personas expertas en igualdad de género y actores procedentes de la sociedad civil (ONG, movimientos sociales, asociaciones, etc.).

${ }^{30} \mathrm{La}$ interseccionalidad como desafío al mainstreaming de género en las politicas públicas. [Revista $V$ asca de Administración Pública, no 87-88, 2010, pp. 225-252]. Ma Ángeles (Maggy) Barrère Unzueta.
} 
Astrolabio. Revista internacional de filosofía Año 2018 Núm. 22. ISSN 1699-7549. pp. 239-251

doi: $10.1344 /$ astrolabio2018.22.20

Humanos, celebrada en Viena en $1993^{31}$. El concepto está recogido en el derecho europeo originario: en el Tratado de Lisboa ${ }^{32}$, y anteriormente en el Tratado de Amsterdam ${ }^{33}$, también está recogido en la Carta de los Derechos Fundamentales de la Unión Europea ${ }^{34}$ y en el TUE ${ }^{35}$. En el derecho derivado podemos encontrarlo en los Programas de Acción Comunitaria $^{36}$ y en un número creciente de regulaciones no vinculantes ${ }^{37}$. En el derecho español, el mainsteaming ha sido recogido en la Ley Orgánica 3/2007 para la igualdad efectiva de mujeres y hombres, en su artículo 15, titulado "Transversalidad del principio de igualdad de trato entre mujeres y hombres" que indica: «El principio de igualdad de trato y oportunidades entre mujeres y hombres informará, con carácter transversal, la actuación de todos los poderes públicos». Este principio ha sido recogido también en el derecho autonómico e informa numerosas políticas públicas.

El mainstreaming se ha incorporado a las políticas sanitarias europeas, como quedó recogido en la Declaración de Madrid de $2001^{38}$, que fue resultado de un Programa promovido por la Oficina Regional Europea de la Organización Mundial de la Salud. La Declaración fue elaborada por participantes de 28 países europeos. En dicha Declaración se acuerdan los principios orientadores de la aplicación del mainstreaming a las políticas sanitarias de los Estados Europeos de la OMS. Añade la Declaración que, aunque muchos Estados de la OMS han aprobado acuerdos internacionales que recogen el género como determinante de la salud, muy pocos han traslado ese compromiso internacional a políticas nacionales concretas. La Declaración expresa que las mujeres y hombres tienen derecho a vivir sin discriminación, incluyéndose la atención sanitaria, la educación y la remuneración laboral. La Organización Mundial de la Salud establece que el disfrute de los niveles más elevados de salud es uno de los derechos fundamentales de todo ser humano sin distinción de raza, religión, creencias políticas, condición económica o social. Para conseguir los mejores niveles de salud es necesario que las políticas

31 Párrafo 36: la igualdad de la mujer será una prioridad para los gobiernos y la ONU, la promoción de la igualdad debería integrarse en las principales actividades de todo el sistema de la ONU.

32 Artículo 8: En todas sus acciones, la Unión se fijará el objetivo de eliminar las desigualdades entre el hombre y la mujer y promover su igualdad.

33 Artículo 2: establece la promoción de la igualdad entre hombres y mujeres como uno de los objetivos de la Unión (junto con la convergencia económica y la promoción del empleo). Artículo 3.2 que obliga a la UE en todas las actividades que desarrolla para cumplir con sus objetivos de tratar de eliminar las desigualdades y a promover la igualdad entre mujeres y hombres.

34 Artículo 23. La igualdad entre hombres y mujeres será garantizada en todos los ámbitos, inclusive en materia de empleo, trabajo y retribución

35 Artículo 2: introducción de la igualdad de género como uno de los objetivos de la Unión. Artículo 3.2: obligación de la UE de tratar de eliminar las desigualdades y de promover la igualdad de género en todas las actividades que desarrolla.

36 Programa de Acción Comunitario sobre igualdad de oportunidades entre hombres y mujeres de la Comisión Europea. ${ }^{37}$ Comunicación de la Comisión 96/67 para incorporar la igualdad de oportunidades para mujeres y hombres en todas las políticas y actividades de la Unión. Recomendación 96/694 sobre la participación equilibrada de mujeres y hombres en el proceso de toma de decisiones.

38 Programa de Mainstreaming de género Oficina Regional Europea de la OMS. La equidad de género en la salud mediante el mainstreaming: La necesidad de avanzar. La Declaración de Madrid. 
Astrolabio. Revista internacional de filosofía Año 2018 Núm. 22. ISSN 1699-7549. pp. 239-251

doi: $10.1344 /$ astrolabio2018.22.20

sanitarias contemplen las diferencias biológicas y los roles de género, pues estos tienen repercusiones sobre las necesidades, los obstáculos y las oportunidades de las mujeres.

La igualdad de género el objetivo del mainstreaming, que supone la ausencia de discriminación basada en el sexo. El género es uno de los determinantes del estado de salud pues los roles sociales asociados a las mujeres son menos valorados que los masculinos y eso da lugar a que los hombres accedan con mayor facilidad a los recursos y tomen el control de los mismos, también incide sobre la toma de decisiones de las mujeres, impidiendo una adecuada protección de la propia salud. El mainstreaming promueve la integración de los condicionantes de género en el diseño de las políticas sanitarias e incluye las llamadas "acciones positivas" orientadas a la consecución de la igualdad. La Declaración insta a los países a implementar programas de formación en género y salud para las personas responsables de las decisiones sanitarias y para los proveedores de salud.

Los Estados Miembros deberán examinar los roles que desempeñan las mujeres y los hombres, el desequilibrio de poder existente en sus relaciones, sus diferentes necesidades, las oportunidades y limitaciones a las que tienen que enfrentarse, y cómo estas determinan diferencias en su exposición a factores de riesgo, en los síntomas, en la gravedad y frecuencia de las enfermedades, en las consecuencias sociales y culturales de la enfermedad y en cómo se crean desigualdades en el acceso a la información, la atención sanitaria y los tratamientos. Se debe fomentar la investigación sobre temas sanitarios con perspectiva de género y los Estados Miembros deberán incluir las perspectivas de género como uno de los criterios para la financiación de cualquier investigación relevante. Esto conlleva establecer directrices y llevar a cabo programas de formación sobre género y salud dirigidos a las personas que investigan y a quienes evalúen las propuestas de investigación. La Declaración también establece que para la eficacia del mainstreaming es indispensable la participación y de todos los sectores relacionados con el sistema de salud, con un papel activo de mujeres y hombres en condiciones de igualdad. Requiere además la incorporación de personas expertas técnicas y políticas. Los Estados deberán asignar los recursos financieros y humanos necesarios para la implementación de las medidas sanitarias sensibles al género.

\section{CONCLUSIONES}

Hemos argumentado que las discriminaciones presentes en una serie de políticas sanitarias tienen repercusión sobre la salud de las mujeres y, en concreto, sobre las afectadas de enfermedades de alta prevalencia femenina. Hay distintos tipos de discriminación por razón de sexo en las políticas sanitarias, de modo que las discriminaciones que hemos mencionado pueden ser clasificadas. María del Mar García Calvente ${ }^{39}$, desarrollando un

\footnotetext{
${ }^{39}$ García Calvente, M. M. (2010). Guía para incorporar la perspectiva de género a la investigación en salud. Eds. María Luisa Jiménez Rodrigo y Emilia Martínez Morante. Granada: Escuela Andaluza de Salud Pública.
} 
Astrolabio. Revista internacional de filosofía Año 2018 Núm. 22. ISSN 1699-7549. pp. 239-251

doi: $10.1344 /$ astrolabio2018.22.20

modelo de Margrit Eichler ${ }^{40}$ clasifica los problemas de sexismo que presentan las políticas e investigaciones sanitarias en tres categorías: androcentrismo, insensibilidad al género y dobles estándares. El primero de estos tres problemas, el androcentrismo, consiste en adoptar una perspectiva masculina, partir de las experiencias de los hombres y equiparar lo masculino a lo humano en general, lo que lleva a construir lo masculino como norma ${ }^{41}$. Del androcentrismo se deriva la invisibilidad de las mujeres y sus problemáticas en la investigación. Encontramos androcentrismo en la realización de ensayos clínicos que no cuentan con mujeres y en el hecho de extrapolar el conocimiento recogido sobre los hombres a las mujeres. También hay androcentrismo en el hecho de que las enfermedades de las mujeres se consideren poco relevantes como objeto de investigación científica o en el hecho de que las enfermedades crónicas (de predominio femenino) se consideren menos importantes que las agudas (de prevalencia masculina). El segundo problema, la insensibilidad al género, desatiende las diferencias entre mujeres y hombres en la salud, no tomando en consideración los condicionantes sociales de la enfermedad (como las relaciones de poder entre los sexos). El tercer problema es el de los dobles estándares que se manifiesta en la exageración o invención de diferencias entre hombres y mujeres, así como la reproducción de estereotipos sexistas. En la endometriosis, encontramos insensibilidad al género en el hecho de que se tilde a las mujeres de débiles, quejicas o histéricas, o en el hecho de que se presuma que acuden al centro de atención primaria para entretenerse.

Podemos añadir algunos otros tipos de discriminación en relación con las enfermedades mencionadas:

- Esencialización biológica de los problemas de salud de las mujeres a la dimensión sexual- reproductiva. Este sesgo reduce a las mujeres a su capacidad reproductora y oculta los aspectos de la salud que no están relacionados con la reproducción. En la endometriosis es frecuente que la enfermedad solo se diagnostique cuando aparece infertilidad. Esto denota que las quejas reiteradas por dolor tienen menos trascendencia social que la afectación a la fertilidad femenina.

- Infrarrepresentación de las mujeres en los ensayos clínicos. El resultado es que sus hallazgos solo pueden ser aplicables a una mitad de la población y las mujeres quedan excluidas del disfrute de los avances científicos. En la endometriosis está situación es especialmente flagrante, pues a pesar de ser una enfermedad femenina se les prescriben fármacos diseñados para patologías de hombres que han recibido mayor atención científica. Falta de perspectiva de género en la recogida de datos estadísticos. En la endometriosis habría que señalar el hecho de no

\footnotetext{
${ }^{40}$ Eichler, M.; Reisman, A. L.; Borins, E. (1992). “Gender Bias in Medical Research”. Women and Therapy, 12(4), United Kingdom: Taylor \& Francis Group, pp. 61-70.

${ }^{41}$ Ortiz, T. (2006). Medicina, historia y género. 130 años de investigación feminista. Oviedo: KRK.
} 
Astrolabio. Revista internacional de filosofía Año 2018 Núm. 22. ISSN 1699-7549. pp. 239-251

doi: 10.1344/astrolabio2018.22.20

mencionarla en los partes de baja, siendo sustituida por una genérica "dismenorrea” (dolor menstrual) que además de resultar prejuiciosa y de desdeñar la gravedad de la enfermedad, no permite la cuantificación estadística. Las afectadas de fibromialgia, al encontrar dificultades para su diagnóstico, también se enfrentan a consecuentes déficits estadísticos.

- Ceguera de género: considerar que la perspectiva de género no aportaría nada significativo al análisis de un problema de salud. Este sesgo es consecuencia de una falta de concienciación en cuestiones de género. Por ejemplo, cuando se acepta que estas enfermedades han sido objeto de escaso interés científico, pero no el hecho de que ello es porque son enfermedades feminizadas.

- Neutralidad de género: Asumir que el hecho de no analizar separadamente a los hombres y a las mujeres prueba que la política no está discriminando por razón de sexo. Por ejemplo, el Real Decreto que regula las enfermedades que son causa de discapacidad ${ }^{42}$ se aplica tanto a hombres como a mujeres que padezcan dichas enfermedades tasadas, de forma indistinta. Sin embargo, no es lo que dice la norma lo que resulta discriminatorio, sino lo que no dice: no figuran las enfermedades altamente incapacitantes de mayor prevalencia femenina.

- Considerar que los testimonios y experiencias de las mujeres tienen un valor meramente anecdótico. Por ejemplo, considerar que las reiteradas quejas de las mujeres con respecto a su deficiente atención sanitaria constituyen una mera anécdota y una fuente irrelevante para una investigación seria.

Lo hasta aquí expuesto permite concluir que es necesario tener en cuenta la perspectiva de las mujeres para efectuar transformaciones políticas sanitarias o de cualquier otra materia. Numerosos problemas deben ser solventados y el feminismo, tanto teórico como activista, constituye un prisma imprescindible para abordar el cambio político de un futuro que pretenda ser justo y garantizar de forma efectiva los derechos fundamentales. Las luchas por la igualdad no han concluido. Todavía queda mucho por hacer y el derecho a la salud aún no ha sido plenamente conquistado por las mujeres.

42 Real Decreto 1971/1999, de 23 de diciembre, de procedimiento para el reconocimiento, declaración y calificación del grado de discapacidad. 